\title{
Pour une nouvelle histoire des professions et des techniques du cinéma
}

Histoire des métiers du cinéma en France avant 1945 | Les producteurs : enjeux créatifs, enjeux financiers | L'auteur de cinéma. Histoire, généalogie, archéologie

\section{Alain P. Michel}

\section{(2) OpenEdition}

1 Journals

\section{Édition électronique}

URL : https://journals.openedition.org/artefact/7300

DOI : $10.4000 /$ artefact.7300

ISSN : 2606-9245

Éditeur :

Association Artefact. Techniques histoire et sciences humaines, Presses universitaires du Midi

\section{Édition imprimée}

Date de publication : 18 juin 2015

Pagination : 239-241

ISBN : 978-2-271-08155-1

ISSN : 2273-0753

\section{Référence électronique}

Alain P. Michel, «Pour une nouvelle histoire des professions et des techniques du cinéma », Artefact

[En ligne], HS 01 | 2015, mis en ligne le 28 avril 2021, consulté le 05 mai 2021. URL : http://

journals.openedition.org/artefact/7300 ; DOI : https://doi.org/10.4000/artefact.7300

Ce document a été généré automatiquement le 5 mai 2021.

\section{(c) (†) $९$}

Artefact, Techniques, histoire et sciences humaines est mise à disposition selon les termes de la Licence Creative Commons Attribution - Pas d'Utilisation Commerciale - Pas de Modification 4.0 International. 


\section{Pour une nouvelle histoire des professions et des techniques du cinéma}

Histoire des métiers du cinéma en France avant 1945 | Les producteurs : enjeux créatifs, enjeux financiers | L'auteur de cinéma. Histoire, généalogie, archéologie

Alain P. Michel

\section{RÉFÉRENCE}

Laurent Le Forestier, Priska Morrissey (dir.), Histoire des métiers du cinéma en France avant 1945, numéro spécial de 1895 : Revue d'histoire du cinéma, n 65, hiver 2011, 264 p. Laurent Creton, Yannick Dehée, Sébastien Layerle, Caroline Moine, Les producteurs : enjeux créatifs, enjeux financiers, Paris, Nouveau Monde Éditions, 2011, 392 p.

Christophe Gauthier, Dimitri Vezyroglou (dir.), L'auteur de cinéma. Histoire, généalogie, archéologie (actes du colloque de Paris, 6-8 décembre 2007), Éditions de l'AFRHC, 2013, $398 \mathrm{p}$.

1 Aujourd'hui, une partie significative du public reste à la fin du film pour regarder le générique alors qu'il ne se passe souvent rien d'autre qu'une présentation de plus en plus longue de noms de personnes et d'institutions. On est passé du « carton » au « déroulant ». Les films des premiers temps se contentaient d'un titre et éventuellement du sigle de la maison de production. Le film Les Temps modernes de Charlie Chaplin (1936) s'ouvrait sur cinquante secondes de credits (générique en anglais). Le troisième volet du Seigneur des anneaux de Peter Jackson ("Le retour du roi », 2003) se termine sur un générique de 9 minutes 33 secondes, soit près de dix fois plus.

Une série de publications récentes, portées par l'Association française de recherche sur l'histoire du cinéma (AFRHC), met en perspective cette évolution de la séance cinématographique et se penche sur les fondements de la mutation dont elle est le 
signe. Ces ouvrages questionnent de façon inédite les dimensions sociotechniques de la création et les conditions concrètes de l'activité cinématographique. Ce décentrement des regards hérite des apports des cultural studies, de l'histoire économique et de la sociologie de l'art. La prise en compte des "minorités » a bousculé l'approche artistique des œuvres. Mais, si, depuis les années 1980, l'ensemble des acteurs de la « fabrique » du film a été remis en lumière, ce collectif est maintenant placé au cœur de la réflexion historienne ${ }^{1}$.

3 Car, derrière le générique, se pose la question de l'« auteur » d'un document hybride qui résulte (presque) toujours du travail d'un groupe. Qui "signe » le film en ouverture et qui est crédité à la fin ? La réponse change avec le temps et n'est pas toujours explicite. Elle prend éventuellement la forme d'une liste hiérarchisée, traduisant plus ou moins clairement un rapport de domination ou un compromis daté entre co-auteurs aux intérêts parfois concordants, mais éventuellement aussi antagonistes. Par ce biais, le spectre de l'analyse cinématographique est élargi depuis l'étude technique des métiers du cinéma jusqu'à l'économie des conventions qui interroge la façon dont les individus impliqués parviennent (dans des situations d'incertitude) à mettre en place des règles de coopération et des normes de comportements.

4 C'est par exemple le «combat " (qui n'est pas seulement juridique ${ }^{2}$ ) entre le producteur - d'abord omnipotent - et le "réalisateur " qui finit pas l'emporter ${ }^{3}$. Sébastien Layerle, Caroline Moine, Laurent Creton et Yannick Dehée montrent que le premier conserve malgré tout un rôle déterminant dans une création qui n'est pas incompatible avec les moyens financiers mobilisés. Christophe Gauthier et Dimitri Vezyroglou soulignent que cette victoire (au moins symboliquement) est le résultat daté d'une construction chaotique et fragile ${ }^{4}$. En cela, le héros " cinéaste ", un temps victorieux, est un modèle historique, une strate archéologique et une figure généalogique. Les mots et le vocabulaire évoluent, les usages sociaux se transforment, les formulations juridiques s'adaptent à des enjeux financiers colossaux dont il faut partager les coûts comme les recettes, par intéressements, sous forme de salaires ou de cachets.

5 La complexité de la question de l'auteur apparaît tout particulièrement autour d'un troisième personnage, le scénariste, dont la place bancale est étudiée par Alain $\mathrm{Carou}^{5}$. Dans les "vues " puis les "tableaux " muets des premiers temps, l'écrivain se contentait de concevoir anonymement une "idée originale » payée au forfait et largement piratée malgré de multiples tentatives de réglementation. Avec l'allongement des films, puis le développement du parlant, émerge un croisement d'intérêts entre les professionnels du texte et les éditeurs de films. Ces derniers ont de plus en plus besoin de la précision du travail du « dialoguiste » et d'une stabilité dans la fabrication de qualité du spectacle cinématographique. L'accord qui en résulte profite de la dispersion syndicale et d'intérêts personnels concordants. L'auteur du texte filmique est rémunéré au nombre de copies diffusées et non selon le principe d'une rémunération proportionnelle aux recettes, comme c'est le cas pour l'écrivain d'une pièce de théâtre. Chacun y trouve son compte. Mais qu'en est-il des autres ?

6 C'est le sujet du numéro spécial de la Revue d'histoire du cinéma. Laurent Le Forestier et Priska Morrissey coordonnent une " histoire des métiers du cinéma en France avant 1945 ", qui fait le point sur la structuration de professions aux limites poreuses (musiciens, monteuses, décorateurs, etc.) qui ont joué un rôle essentiel dans le 
développement d'un secteur d'activité en mutation. Dans le contexte du passage d'une forme d'artisanat à une espèce d'industrie, les hiérarchies sont bouleversées, les fonctions sont distinguées et se divisent, mais tardent à être reconnues, de même que se brouillent les apports techniques et artistiques de chacun ${ }^{6}$. Il en ressort que la perception des métiers du cinéma évolue en quatre périodes. D'abord l'émergence de postes techniques spécifiques d'une activité foraine (1895-1910), marquée par l'œuvre de Georges Méliès. Suit une phase d'opacité (1910-1930), au cours de laquelle le travail des professionnels du cinéma se développe, mais reste occulte afin de ne pas contrarier la visée " artistique » des maisons de production. Au contraire, le développement du cinéma " parlant » correspond à une phase d'affirmation (1930-1950), qui voit les métiers s'organiser (syndicats, formation, festival, etc.) en réaction avec la concurrence du cinéma américain. La technique (française) devient un gage de qualité et une garantie d'« artisticité » des films ${ }^{7}$. La dernière période est celle du reflux (1960-1990), à l'époque de "la politique des auteurs " et de la cinéphilie triomphante. Les témoignages et les récits professionnels des grands collaborateurs viennent toutefois atténuer cette image du réalisateur comme seul auteur. Cette évolution instable permet de mieux comprendre qu'aujourd'hui, le succès des séries et la pression des " saisons " bousculent la hiérarchie de la fabrique des films.

7 Cette série d'ouvrages sort durablement l'histoire du cinéma des ornières de la seule appréciation esthétique des films (de fiction) et permet de dépasser la dichotomie stérile entre art et industrie. Ainsi, la technique et les techniciens sont remis historiquement sur le devant de la scène.

\section{NOTES}

1. Christian Delage (dir.), La fabrique des images contemporaines, Paris, Éditions Cercle d'art, 2007, 189 p. (textes de Christian Delage, Vincent Guigueno et André Gunthert). Pour une dimension plus sociologique, voir Kristian Feigelson, La fabrique filmique : métiers et professions, Paris, Armand Colin, 2011 ; Gwenaële Rot, Laure de Verdalle (dir.), Le cinéma : travail et organisation, Paris, la Dispute, 2013, 236 p.

2. Jean-Pierre Jeancolas et al., L'auteur du film. Description d'un combat, Arles-Lyon, Actes Sud-Institut Lumière, 1996.

3. Laurent Creton, Yannick Dehée, Sébastien Layerle, Caroline Moine, Les producteurs : enjeux créatifs, enjeux financiers, Paris, Nouveau Monde Éditions, 2011.

4. Christophe Gauthier, Dimitri Vezyroglou (dir.), L'auteur de cinéma. Histoire, généalogie, archéologie, Éditions de l'AFRHC, 2013, p. 12-13.

5. Alain Carou, "Le scénario français en quête d'auteurs (1908-1918) ", dans Laurent Le Forestier, Priska Morrissey (dir.), Histoire des métiers du cinéma en France avant 1945, 1895 : Revue d'histoire du cinéma, $\mathrm{n}^{\circ}$ 65, 2011, p. 28-51 ; Alain Carou, " De l'usage de l'idée d'auteur dans l'histoire de la production cinématographique en France (1908-1937) ", dans Christophe Gauthier, Dimitri Vezyroglou (dir.), L'auteur de cinéma..., op. cit., p. 59-68.

6. Laurent Le Forestier, Priska Morrissey (dir.), Histoire des métiers du cinéma en France avant 1945, numéro spécial de 1895 : Revue d'histoire du cinéma, n 65, hiver 2011, p. 12. 
7. Ibid., p. 16.

\section{AUTEURS}

ALAIN P. MICHEL

Université d'Evry-Val d'Essonne, Laboratoire d'histoire économique, sociale et des techniques (LHEST, EA 4525) 